\title{
Fuzzy Comprehensive Evaluation of Search Engine Model for Online
}

\author{
Xiaohui $\mathrm{Li}^{1, \mathrm{a}}$, Jianqiao $\mathrm{Yu}^{2, \mathrm{~b}}$ and Yifeng $\mathrm{Dou}^{3, \mathrm{c}}$ \\ ${ }^{1,2,3}$ College of Computer and Information Science, Southwest University, Chongqing, China

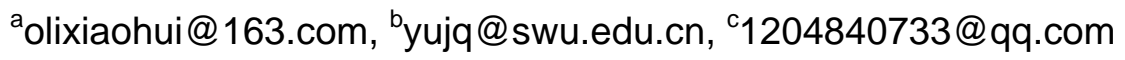

Keywords: Search engine, Fuzzy comprehensive evaluation, Analytic hierarchy process

\begin{abstract}
With the rapid development of Internet, the search engine becomes an important tool for searching information gradually. In order to choose an appropriate and reliable search engine, a research on search engine evaluation has been a hot topic. In this paper, we adopt a fuzzy comprehensive evaluation method by computing indicator weight and building fuzzy matrix to evaluate the search engines.
\end{abstract}

\section{Introduction}

With the popularization and development of Internet, people is accustomed to gaining information by use the search engine. However, owing to variety of search engines and complexity of the content, making the selection of search engine has become a hot topic [1]. Therefore, building a mathematical model and obtaining the ranking through analyzing the search engine has a big practical significance. This article using fuzzy comprehensive evaluation method to conduct a proper evaluation of the search engines.

\section{The evaluation index system for search engine}

In numerous evaluation index systems, the flow and velocity index, the retrieval performance index, the service function index are the core indexes which used to detect the performance of search engine, these indexes are the basic function of the Internet search engine and represent the minimum requirement of search engine evaluation.

Understand the situation of search engine at home and abroad by literature review, this article summarizes the main indexes in accordance with the operation habits of users [2].

(1) The flow and velocity index, the main indexes are the web traffic rank, click rate and access speed.

(2) The retrieval performance index mainly includes precision ratio and recall ratio.

(3) The service function index mainly includes user interface and user experience evaluation.

\section{Fuzzy comprehensive evaluation of search engine model}

The method of fuzzy comprehensive evaluation is a new method which apply the fuzzy mathematics to judge the system merits. There are multiple perspectives should be assessed for the integrated ranking of search engine [3]. In this paper, seven major domestic search engines are selected, including Baidu, Sogou, Google China, Sou 360, Yahoo China, Soso, Iask.

(1) Establishment of factor set and the hierarchical structure

The judgement factors are the judgement indexes, set the factor set as $U=\left\{u_{1}, u_{2}, \cdots, u_{i}, \cdots, u_{n}\right\}$, in which $u_{i}=(1,2, \ldots, n)$ is the i-th judgement index. There are many factors should be considered in the Internet search engine evaluation. Most methods can only analyze the Internet search engine performance from qualitative and quantitative aspects, and Analytic Hierarchy Process (AHP) is a combination of qualitative and quantitative, systematic and hierarchical analysis method [4].

The hierarchical structure is established in this paper as shown in Fig. 1.

(2) Determine the evaluation matrix 


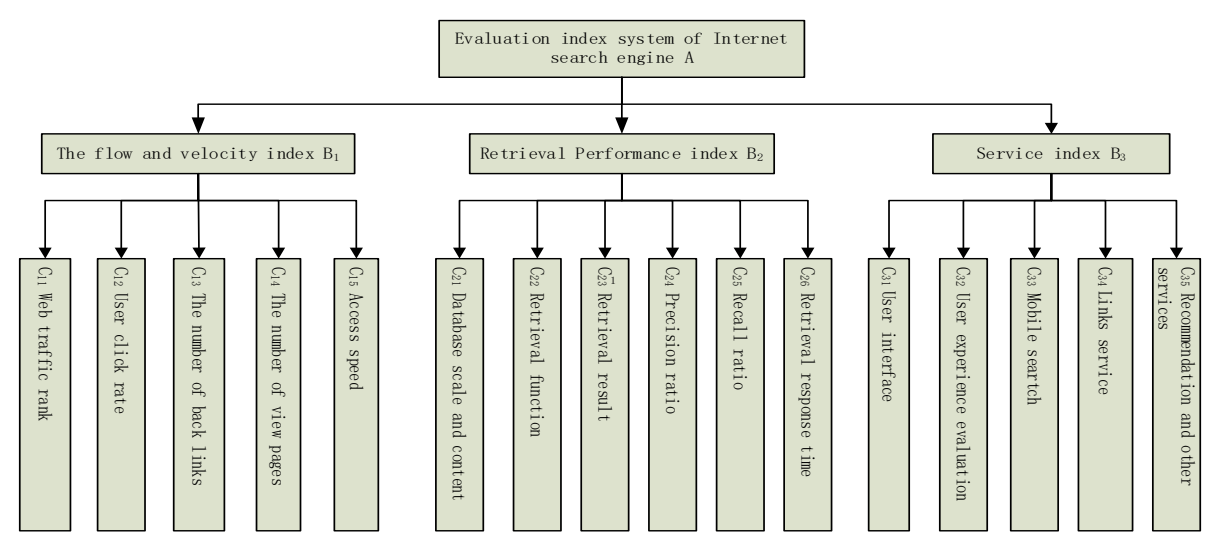

Figure 1. The hierarchical structure of the fuzzy comprehensive evaluation of search engine Firstly, the single factor evaluation of $u_{i}(i=1,2, \ldots, n)$ is made in $U$, and the membership degree $r_{i j}$ which is $u_{i}$ to evaluation grade $v_{j}(j=1,2,3,4,5)$ is determined. So the evaluation set $\mathrm{r}_{i j}=\left(\mathrm{r}_{i 1}, r_{i 2}, r_{i 3}, r_{i 4}, r_{i 5}\right)$ of the $\mathrm{i}$-th single factor evaluation $u_{i}$ is obtained [5].

The general evaluation matrix as shown in Eq. 1.

$$
R=\left[\begin{array}{ccccc}
r_{11} & r_{12} & r_{13} & r_{14} & r_{15} \\
r_{21} & r_{22} & r_{23} & r_{24} & r_{25} \\
r_{11} & r_{i 2} & r_{i 3} & r_{i 4} & r_{i 5} \\
\vdots & \vdots & \vdots & \vdots & \vdots \\
r_{n 1} & r_{n 2} & r_{n 3} & r_{n 4} & r_{n 5}
\end{array}\right] .
$$

To quantitative study the influencing level of each factor will brings some ambiguity error. Here, the concept of the weight is introduced, $W=\left\{\omega_{1}, \omega_{2}, \ldots, \omega_{i}, \ldots, \omega_{n}\right\}$, in which $W$ represents the weight set, and $\omega_{\mathrm{i}}$ represents the weight of $u_{i}$, which is the degree of $u_{i}$ in the general assessment.

(3) Calculation weight

Firstly, it should be distributed reasonable weights for each factor, takes AHP method to match and compare with every factor, and then judge the relative importance degree between each factor. Finally, calculate feature values of comparison matrix pairs to achieve the weight vectors.

In detail, the steps of weights calculation are following:

Step 1. Construction of comparison matrix pairs

From the second layer of the Hierarchical model, the paired comparison method is used for the same layer factors which are subject to each factor layer until the lowest level to constitute pairwise comparison matrix. The judgment matrix is established as shown in Eq. 2 and criteria scale represents the relative importance dimensions of $B_{i}$ to $B_{j}$ in $B_{i j}$. Criteria scale is a quantitative analysis and the numerical value of $B_{i j}$ is defined according to the rules as shown in Table 1.

$$
B=\left[\begin{array}{llll}
B_{11} & B_{12} & B_{13} & B_{14} \\
B_{21} & B_{22} & B_{23} & B_{24} \\
B_{31} & B_{32} & B_{33} & B_{34} \\
B_{41} & B_{42} & B_{43} & B_{44}
\end{array}\right] .
$$

Step 2. Calculate weight of the matrix

According to AHP, this paper obtains the feature vectors by solving a positive reciprocal matrix characteristic value, and the weight vector is achieved after normalized, $Q_{k}=\left[q_{1 k}, q_{2 k}, \ldots, q_{k k}\right]^{T}$, in which $q_{i k}$ is the relative weight of $B_{i}$ to the factor in higher layer. According to $\mathrm{A}-\lambda \mathrm{I}=0$, calculate maximum eigenvalue $\lambda_{\max }$ and corresponding characteristic vector by using MATLAB 2013b software. The calculation formula is as shown in Eq. 3.

$$
\omega_{i}=\frac{\sum_{j=1}^{n} B_{i j}}{\sum_{k=1}^{n} \sum_{j=1}^{n} B_{k j} k} .
$$


Table 1. Criteria scale

\begin{tabular}{cc}
\hline Criteria scale & defined rules \\
\hline 1 & $B_{i}$ and $B_{j}$ is equally important \\
3 & $B_{i}$ is a little important than $B_{j}$ \\
5 & $B_{i}$ is obviously important than $B_{j}$ \\
7 & $B_{i}$ is strongly important than $B_{j}$ \\
9 & $B_{i}$ is extremely important than $B_{j}$ \\
$2,4,6,8$ & Between the two adjacent criteria scale \\
above Non-zero countdown & if $B_{i}$ has a number above for $B_{j}, B_{j}$ has countown for $B_{i}$ \\
\hline
\end{tabular}

Step 3. Consistency test

For the judgment matrix is the basis for calculating the weight, the matrix should be largely consistent, therefore, it is important to analysis compatibility and error of judgement matrices. In order to measure the reliability of judgment, consistency index (C.I.) is calculated, C.I. $=\frac{\lambda_{\max }-n}{n-1}$, in which $\lambda_{\max }$ represents the maximum eigenvalue of matrix $B, \mathrm{n}$ indicates the reciprocal matrices order, the smaller of C.I., the higher reliability of the weight.

The Table 2 details the mean random consistency index (R.I.) which is calculated 1000 times by the 1-12 reciprocal matrix. Calculate the consistency ratio (C.R.), C.R. $=\frac{\text { C.I. }}{\text { R.I. }}$, in general, if C.R. < 0.1 , the judgment is satisfactory and compatibility, the calculated value $\omega$ is acceptable, on this condition the reciprocal matrix is called consistency matrix, then return to Step 4, otherwise the reciprocal matrix should be revise and return to Step2.

Table 2. The mean random consistency index

\begin{tabular}{|c|c|c|c|c|c|c|c|c|c|c|c|c|}
\hline Order & 1 & 2 & 3 & 4 & 5 & 6 & 7 & 8 & 9 & 10 & 11 & 12 \\
\hline R.I. & 0 & 0 & 0.58 & 0.89 & 1.12 & 1.26 & 1.36 & 1.41 & 1.46 & 1.49 & 1.52 & 1.54 \\
\hline
\end{tabular}

Based on the above theory, the pairwise comparison matrices are established on search engine in all aspects including evaluation target level and criteria level, flow and velocity and sub rule layer, and so on. The weight of each indicator is calculated, and the consistency of acquired data is judged. Table 3. The judgement matrix between target layer and rule layer in the evalutation index system of Internet search engine. A-B

\begin{tabular}{lllllc}
\hline $\mathrm{A}$ & $\mathrm{B}_{1}$ & $\mathrm{~B}_{2}$ & $\mathrm{~B}_{3}$ & weight & consistency test \\
\hline $\mathrm{B}_{1}$ & 1 & $1 / 3$ & $1 / 4$ & 0.1669 & $\lambda_{\max }=2.5527$ \\
$\mathrm{~B}_{2}$ & 3 & 1 & 2 & 0.6099 & C.I. $=0.0000$, R.I. $=0.5800$ \\
$\mathrm{~B}_{3}$ & $1 / 4$ & $1 / 2$ & 1 & 0.2232 & C.R. $=0.0000$ \\
\hline
\end{tabular}

Table 4. The juding maritix between traffic,speed and sub-rule layer. $\mathrm{B}_{1}-\mathrm{C}_{1 \mathrm{j}}$

\begin{tabular}{llllllll}
\hline $\mathrm{B}_{1}$ & $\mathrm{C}_{11}$ & $\mathrm{C}_{12}$ & $\mathrm{C}_{13}$ & $\mathrm{C}_{14}$ & $\mathrm{C}_{15}$ & weight & consistency test \\
\hline $\mathrm{C}_{11}$ & 1 & 4 & 2 & 4 & 3 & 0.4054 & $\lambda_{\max }=5.0882$ \\
$\mathrm{C}_{12}$ & $1 / 4$ & 1 & $1 / 3$ & 1 & $1 / 2$ & 0.0850 & C.I. $=0.0221$ \\
$\mathrm{C}_{13}$ & $1 / 2$ & 3 & 1 & 4 & 3 & 0.2916 & R.I. $=1.1200$ \\
$\mathrm{C}_{14}$ & $1 / 4$ & 1 & $1 / 4$ & 1 & $1 / 2$ & 0.0803 & C.R. $=0.0197$ \\
$\mathrm{C}_{15}$ & $1 / 3$ & 2 & $1 / 3$ & 2 & 1 & 0.1377 & \\
\hline
\end{tabular}

Step 4. Order of hierarchy

From tables in Step 3 shows that, relative to the pros and cons of the Internet search engine, The rank of each index important listed in flow and velocity in criteria level is as follows: web traffic rank $>$ reverse link number $>$ access speed $>$ users click rate $>$ browse page number, the rank in 
retrieval performance is: precision ratio $>$ retrieval result $>$ retrieval response time $>$ database scale and content $>$ recall ratio $>$ retrieval function, and the order in the service function is: user interface $=$ user experience evaluation $>$ blogroll Service $>$ mobile search $>$ recommendation and other services.

The comprehensive sequence is ranked by using the calculation results of single level sequencing. According to the above theory, the comprehensive ranking results are calculated as shown in Table 7.

Table 5. The juding maritix between retivetal perfermance and sub-rule layer $B_{2}-C_{2 j}$

\begin{tabular}{lllllllll}
\hline $\mathrm{B}_{2}$ & $\mathrm{C}_{21}$ & $\mathrm{C}_{22}$ & $\mathrm{C}_{23}$ & $\mathrm{C}_{24}$ & $\mathrm{C}_{25}$ & $\mathrm{C}_{26}$ & weight & consistency test \\
\hline $\mathrm{C}_{21}$ & 1 & 1 & $1 / 2$ & $1 / 3$ & 1 & 2 & 0.1305 & $\lambda_{\max }=6.2296$ \\
$\mathrm{C}_{22}$ & 1 & 1 & $1 / 3$ & $1 / 2$ & 1 & $1 / 2$ & 0.1023 & C.I. $=0.0459$ \\
$\mathrm{C}_{23}$ & 2 & 3 & 1 & $1 / 2$ & 2 & 2 & 0.2296 & R.I. $=1.2600$ \\
$\mathrm{C}_{24}$ & 3 & 2 & 2 & 1 & 2 & 2 & 0.2947 & C.R. $=0.0364$ \\
$\mathrm{C}_{25}$ & 1 & 1 & $1 / 2$ & $1 / 2$ & 1 & 1 & 0.1184 & \\
$\mathrm{C}_{26}$ & $1 / 2$ & 2 & $1 / 2$ & $1 / 2$ & 1 & 1 & 0.1244 & \\
\hline
\end{tabular}

Table 6. The juding maritix between service function and sub-rule layer $B_{3}-C_{3 j}$

\begin{tabular}{llllllll}
\hline $\mathrm{B}_{3}$ & $\mathrm{C}_{31}$ & $\mathrm{C}_{32}$ & $\mathrm{C}_{33}$ & $\mathrm{C}_{34}$ & $\mathrm{C}_{35}$ & weight & consistency test \\
\hline $\mathrm{C}_{31}$ & 1 & 1 & 3 & 3 & 4 & 0.3382 & $\lambda_{\max }=5.0851$ \\
$\mathrm{C}_{32}$ & 1 & 1 & 3 & 3 & 4 & 0.3382 & C.I. $=0.0213$ \\
$\mathrm{C}_{33}$ & $1 / 3$ & $1 / 3$ & 1 & $1 / 2$ & 2 & 0.1081 & R.I. $=1.1200$ \\
$\mathrm{C}_{34}$ & $1 / 3$ & $1 / 3$ & 2 & 1 & 2 & 0.1434 & C.R. $=0.0190$ \\
$\mathrm{C}_{35}$ & $1 / 4$ & $1 / 4$ & $1 / 2$ & $1 / 2$ & 1 & 0.0722 & \\
\hline
\end{tabular}

Table 7. Hierarchy comprehensive order

\begin{tabular}{|c|c|c|c|c|c|}
\hline $\begin{array}{c}\text { Target } \\
\text { layer (A) }\end{array}$ & $\begin{array}{l}\text { Criteria } \\
\text { layer (B) }\end{array}$ & Weight & Sub-criteria layer (C) & Weight & $\begin{array}{l}\text { Combined } \\
\text { Weights }\end{array}$ \\
\hline \multirow{16}{*}{$\begin{array}{l}\text { Evaluation } \\
\text { index } \\
\text { system of } \\
\text { Internet } \\
\text { search } \\
\text { engine (A) }\end{array}$} & \multirow{5}{*}{$\begin{array}{l}\text { Flow and } \\
\text { velocity } \\
\text { index (B1) }\end{array}$} & \multirow{5}{*}{0.1669} & Web traffic rank ((C11) & 0.4054 & 0.0677 \\
\hline & & & User click rate (C12) & 0.085 & 0.0142 \\
\hline & & & The number of back links (C13) & 0.2916 & 0.0487 \\
\hline & & & The number of view pages (C14) & 0.0803 & \\
\hline & & & Access speed (C15) & 0.1377 & 0.0230 \\
\hline & \multirow{6}{*}{$\begin{array}{c}\text { Retrieval } \\
\text { performan } \\
\text { ce index } \\
\text { (B2) }\end{array}$} & \multirow{6}{*}{0.6099} & Database scale and content (C21) & 0.1305 & 0.0796 \\
\hline & & & Retrieval function (C22) & 0.1023 & 0.0624 \\
\hline & & & Retrieval result (C23) & 0.2296 & 0.1400 \\
\hline & & & Precision ratio (C24) & 0.2947 & \\
\hline & & & Recall ratio (C25) & 0.1184 & 0.0722 \\
\hline & & & Retrieval response time (C26) & 0.1244 & 0.0759 \\
\hline & \multirow{5}{*}{$\begin{array}{c}\text { Service } \\
\text { index (B3) }\end{array}$} & \multirow{5}{*}{0.2232} & User interface (C31) & 0.3382 & 0.0755 \\
\hline & & & User experience evaluation (C32) & 0.3382 & 0.0755 \\
\hline & & & Mobile search (C33) & 0.1081 & 0.0241 \\
\hline & & & Links service (C34) & 0.1434 & \\
\hline & & & $\begin{array}{l}\text { Recommendation and other } \\
\text { services (C35) }\end{array}$ & 0.0722 & 0.0161 \\
\hline
\end{tabular}


(4) Establishment of evaluation grade and evaluation set

This paper uses 5 level evaluation, and set the evaluation set to $V=\{$ very good, good, general, poor, very poor $\}=\{95,75,55,35,15\}$. According to the corresponding evaluation scale, the membership matrix of evaluation index set is obtained by statistics expert scoring, as shown in Table 8.

(5) Construction of the Internet search evaluation model

Above we get evaluation matrix $R$, weight set $W$ and evaluation set $V, B=R^{*} W=\left(b_{1}, b_{2}, b_{3}, b_{4}, b_{5}\right)$. The result of fuzzy comprehensive evaluation is a vector with five components which is corresponding to the following five grades of membership degree including poor, relatively poor, normal, relatively good, and good. The results by fuzzy transformation generally does not meet needs of evaluation, therefore, it is necessary to normalize for $B$. $B$ ' could be calculated by MATLAB2013b, and $b^{\prime}=\frac{b_{j}}{\sum_{j=1}^{5} b_{j}}$.So far the first level evaluation model has been established, the analytical model of the second and third levels is based on the calculated results of the first level model, the solution process by using MATLAB as shown Eq. 4, Eq. 5, Eq. 6, Eq.7

Table 8. Fuzzy evaluation set of Internet search engine

\begin{tabular}{|c|c|c|c|c|c|c|c|c|}
\hline \multicolumn{2}{|c|}{$\begin{array}{l}\text { Target layer Criteria layer } \\
\begin{array}{ll}\text { (A) } & \text { (B) }\end{array}\end{array}$} & \multirow[t]{2}{*}{ Weight } & Sub-criteria layer (C) & Weight & \multicolumn{4}{|c|}{$\begin{array}{l}\text { Evaluation set } \\
\quad(\mathrm{V}=16) \\
\mathrm{R}_{1} \mathrm{R}_{2} \mathrm{R}_{3} \mathrm{R}_{4} \mathrm{R}_{5} \\
\end{array}$} \\
\hline \multirow{16}{*}{$\begin{array}{l}\text { Evaluation } \\
\text { index } \\
\text { system of } \\
\text { Internet } \\
\text { search } \\
\text { engine (A) }\end{array}$} & \multirow{5}{*}{$\begin{array}{l}\text { Flow and } \\
\text { velocity } \\
\text { index (B1) }\end{array}$} & & Web traffic rank (C11) & 0.4054 & 4 & 6 & 7 & 51 \\
\hline & & \multirow{4}{*}{0.1669} & User click rate (C12) & 0.0850 & 5 & 8 & 4 & 60 \\
\hline & & & The number of back links (C13) & 0.2916 & 4 & 6 & 7 & 51 \\
\hline & & & The number of view pages (C14) & 0.0803 & 5 & 8 & 5 & 32 \\
\hline & & & Access speed (C15) & 0.1377 & 4 & 6 & 6 & 52 \\
\hline & \multirow{7}{*}{$\begin{array}{c}\text { Retrieval } \\
\text { performance } \\
\text { index (B2) }\end{array}$} & \multirow{7}{*}{0.6099} & Database scale and content (C21) & 0.1305 & 5 & 7 & 6 & 50 \\
\hline & & & Retrieval function (C22) & 0.1023 & 6 & 6 & 6 & 32 \\
\hline & & & Retrieval result (C23) & 0.2296 & 7 & 8 & 6 & 20 \\
\hline & & & Precision ratio (C24) & 0.2947 & 4 & 9 & 8 & 20 \\
\hline & & & Recall ratio (C25) & 0.1184 & 3 & 7 & 6 & 52 \\
\hline & & & Retrieval response time (C26) & 0.1244 & 6 & 7 & 5 & 50 \\
\hline & & & User interface (C31) & 0.3382 & 4 & 6 & 6 & 52 \\
\hline & \multirow{4}{*}{$\begin{array}{l}\text { service index } \\
\text { (B3) }\end{array}$} & \multirow{4}{*}{0.2232} & User experience evaluation (C32) & 0.3382 & 2 & & 8 & 72 \\
\hline & & & Mobile search (C33) & 0.1081 & 2 & & 7 & 72 \\
\hline & & & Links service (C34) & 0.1434 & 1 & & 9 & 72 \\
\hline & & & $\begin{array}{l}\text { Recommendation and other services } \\
\text { (C35) }\end{array}$ & 0.0722 & 0 & & 9 & 83 \\
\hline
\end{tabular}

$$
\begin{aligned}
& \left|\begin{array}{lllll}
0.1739 & 0.2609 & 0.3043 & 0.2174 & 0.0435
\end{array}\right| \\
& \begin{array}{lllll}
0.2174 & 0.3478 & 0.1739 & 0.2609 & 0.0000
\end{array} \\
& B_{1}=A_{1}^{*} R_{1}=\left(\begin{array}{lllll}
0.4054 & 0.0850 & 0.2916 & 0.0803 & 0.1377
\end{array}\right)\left(\begin{array}{llllll}
0.1739 & 0.2609 & 0.3043 & 0.2174 & 0.0435
\end{array}=\left(\begin{array}{lllll}
0.1811 & 0.2753 & 0.2803 & 0.2141 & 0.0493
\end{array}\right)\right. \\
& \begin{array}{llllll}
0.2174 & 0.3478 & 0.2174 & 0.1304 & 0.0870
\end{array}
\end{aligned}
$$

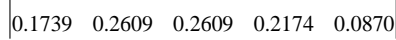

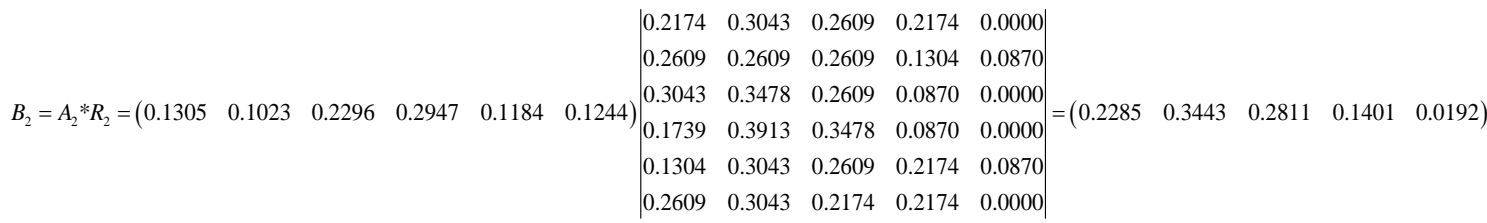

$$
\begin{aligned}
& \left|\begin{array}{lllll}
0.1739 & 0.2609 & 0.2609 & 0.2174 & 0.0870
\end{array}\right| \\
& \begin{array}{lllll}
0.0870 & 0.1739 & 0.3478 & 0.3043 & 0.0870
\end{array}
\end{aligned}
$$

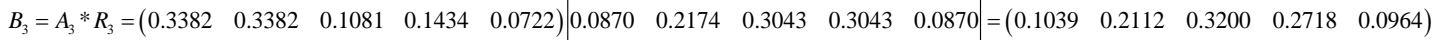

$$
\begin{aligned}
& \begin{array}{lllll}
0.0435 & 0.1739 & 0.3913 & 0.3043 & 0.0870
\end{array} \\
& \begin{array}{lllll}
0.0000 & 0.2174 & 0.3478 & 0.2609 & 0.2174
\end{array} \mid
\end{aligned}
$$


(7)

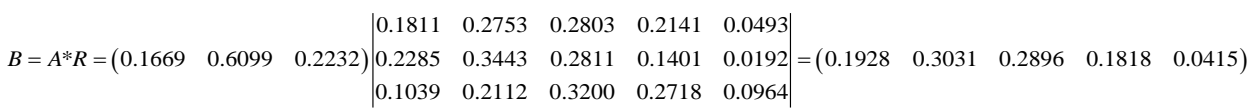

Combined with 3.4, we can make the following matrix transformation:

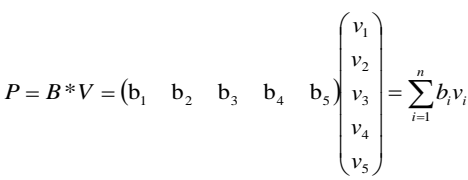

So

$P=B^{*} V=\left(\begin{array}{lllll}0.1928 & 0.3031 & 0.2896 & 0.1818 & 0.0415\end{array}\right)\left[\begin{array}{c}95 \\ 75 \\ 55 \\ 35 \\ 15\end{array}\right]=63.9620$

(6) Results analysis

According to $P=B^{*} V$, we can get $P=63.9620, P_{1}=62.5734, P_{2}=59.7880, P_{3}=61.2238$, $P_{4}=61.3735, P_{5}=60.3314, P_{6}=57.9834$. The higher $\mathrm{P}$, the higher performance of the search engine. According to above computation achieve the ranking: Baidu $>$ Sogou $>$ Yahoo China $>$ Sou $360>$ Google China $>$ Soso $>$ Iask.

1) Results analysis of the flow and velocity

The website speed affects the user experience directly and many reasons lead to the site of long buffer time and slow response speed, such as poor performance, too many cache pictures etc.

2) Results analysis of retrieval performance

In order to improve the retrieval function, detailed information should be provided to help the user, and furthermore improve the natural language understanding ability to reduce the burden on users.

3) Results analysis of service function

The evaluation results show that organizing the network information resources scientifically and the unification of classification and theme languages can improve the recall ratio and precision ratio.

\section{Conclusion}

With the rapid development of the Internet, the search engine has developed greatly, which makes indicators' value is fuzzy, uncertainty, random even unknown, this paper adopts AHP to achieve the index weights based on the establishment of evaluation index, finally obtains the rank of seven major domestic search engines. This paper gives an evaluation means of fuzzy comprehensive evaluation method for the Internet search engine, but with the development of search engine, the focus will change, and the perfect evaluation index and weight index brings great help to correct evaluation.

\section{References}

[1] Chen, J. (2010). A Flexible Fuzzy Comprehensive Evaluation Method.Intelligent Information Technology and Security Informatics, International Symposium on (pp.502-506). IEEE.

[2] Liu, Z. C., \& Jiang, F. K. (2004). The fuzzy comprehensive evaluation on the performance of search engine. Mathematics in Practice \& Theory.

[3] Ling-Juan, L. I., \& Shen, L. T. (2006). An improved multilevel fuzzy comprehensive evaluation algorithm for security performance. Journal of China Universities of Posts \& Telecommunications, 13(4), 48-53.

[4] Li, H., \& Qiang, M., \& Mei, Y. L., \& Min, M. (2004). Analysis and Study on AHP-Fuzzy Comprehensive Evaluation, China Safety Science Journal, 14(7), 86-89.

[5] Guo, X. D., \& Fu, X. X. (2003). Improvement of fuzzy comprehensive evaluation method. Journal of Chongqing University. 